$$
\text { CONF-960543-15 }
$$

NO INTERNET DISTRIBUTION UCRL-JC-116323

PREPRINT

\title{
Use of Thin Wall Imaging in the Diagnosis of Laser Heated Hohlraums
}

L. J. Suter, A. R. Thiessen, F. Ze,

R. Kauffman, R. H. Price, V. C. Rupert,

V. W. Slivinsky, and C. Wang

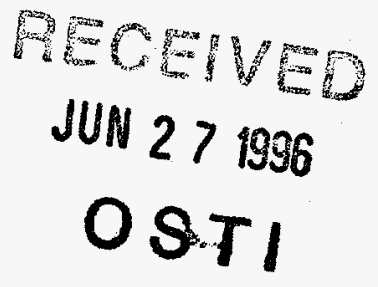

This paper was prepared for submittal to the 11th Topical Conference on High Temperature Plasma Diagnostics Monterey, California

May 12-16, 1996

May 14, 1996
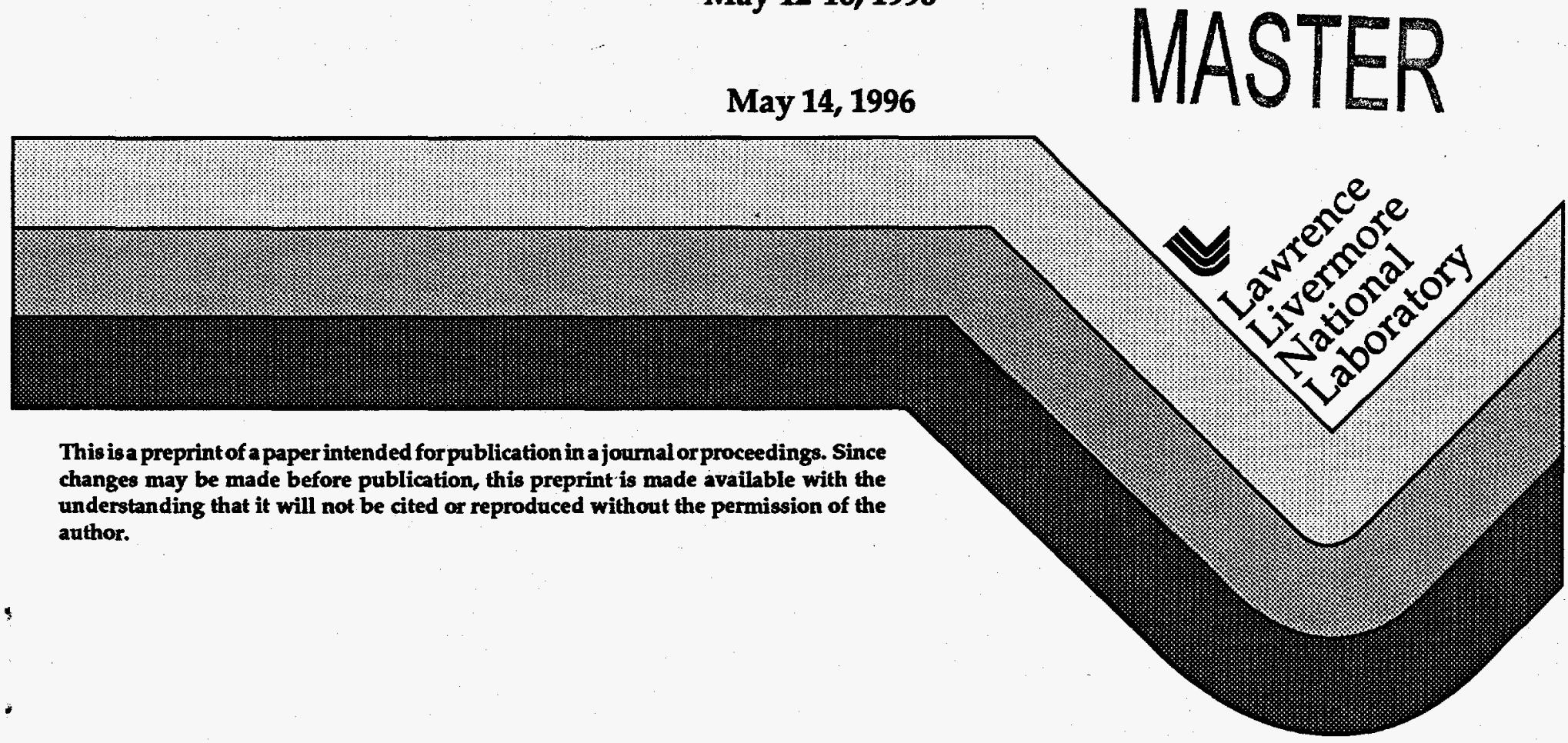
This document was prepared as an account of work sponsored by an agency of the United States Government. Neither the United States Government nor the University of California nor any of their employees, makes any warranty, express or implied, or assumes any legal liability or responsibility for the accuracy, completeness, or usefulness of any information, apparatus, product, or process disclosed, or represents that its use would not infringe privately owned rights. Reference herein to any specific commercial product, process, or service by trade name, trademark, manufacturer, or otherwise, does not necessarily constitute or imply its endorsement, recommendation, or favoring by the United States Government or the University of California. The views and opinions of authors expressed herein do not necessarily state or reflect those of the United States Government or the University of California, and shall not be used for advertising or product endorsement purposes. 
Use of thin wall imaging in the diagnosis of laser heated hohlraums

L. J. Suter, A. R. Thiessen, F. Ze, R. Kauffman, R. H. Price, V. C. Rupert, V. W. Slivinsky, C. Wang

Lawrence Livermore National Laboratory

High-Z, laser heated hohlraums can be made thick enough to contain thermal radiation, yet thin enough to let out xrays $>\sim 6 k e V$ produced by hot, relatively dense blow-off plasma. We use such "thin wall hohlraums" to observe the physical location of hot, dense, laser produced hohlraum plasmas. This technique has allowed us to come to some understanding of laser transport/deposition, plasma stagnation and bulk plasma filling.

Thin wall imaging is an experimental technique which can provide information about hot plasma inside a laser heated hohlraum. For fabrication ease, typical hohlraums used for Nova [1] ICF experiments are made of 25 micron thick gold. However, for an ordinary ICF experiment only $\sim 2$ microns of gold is actually needed to contain the thermal x-rays. Consequently, specially made hohlraums of 2 micron thick gold should perform like thicker hohlraums, as far as the thermal radiation is concerned, but allow more energetic radiation, $>6-8 \mathrm{keV}$ to escape. We can image this radiation and interpret it in various manners.

The earliest work on thin wall imaging suggested that approximately images of $\sim>6 \mathrm{keV}$ emission would provide a map of laser deposition inside a hohlraum. This view came from our understanding of laser heated discs. At the surface of a laser heated disc there are steep 
temperature and density gradients of opposite signs. At ICF irradiances, $\sim 10^{14}$ to $10^{15} \mathrm{w} / \mathrm{cm}^{2}$, the temperature rises to several $\mathrm{keV}$ and the density drops to $<10^{21} / \mathrm{cc}$ over a distance $<\sim 100$ microns. Beyond this, the temperature approximately plateaus while the density continues to drop. Qualitatively, we would expect $>6 \mathrm{keV}$ emission will peak towards the hot end of this region of steep gradients since it requires both temperature (a few $\mathrm{keV}$ or more) and density to produce $>6 \mathrm{keV}$ radiation. The non-LTE, XSN [2] average atom model provides us with a quantitative functional dependence in the temperature and density regime of interest. For gold, a good fit to the XSN estimates of $>6 \mathrm{keV}$ emission is

$$
\text { emission/cc }=7 \mathrm{e} 17 \quad \mathrm{~T}^{4.05} \mathrm{\rho}^{2.4} \quad[1]
$$

The units are $w / \mathrm{cm}^{3}, \mathrm{keV}$ and $\mathrm{g} / \mathrm{cc}$. This expression is valid for $1 \mathrm{keV}<\mathrm{T}_{\mathrm{e}}<5 \mathrm{keV}$ and $0.001<\rho<0.03 \mathrm{~g} / \mathrm{cc}$. Above $5 \mathrm{keV}$ the emission rises more slowly. Below $5 \mathrm{keV}$, more rapidly.

In a disc simulations with the Lasnex [3] code, the peak of this emission essentially coincides with the peak laser deposition. Because of this coincidence, thin wall imaging should provide a map of laser deposition at early times when the hohlraum plasma is disc-like. However, at later times, when plasma accumulates in the hohlraum, the $>6 \mathrm{keV}$ images can be very different from an "image" of laser deposition. 
In 1981 we performed our first thin wall imaging experiments with the Shiva laser [4]. These experiments used $2.5 \mathrm{~mm}$ diameter spherical hohlraums with 900 micron diameter laser entrance holes. We irradiated these hohlraums with $4 \mathrm{kj}$ of 1 micron light in a 900ps FWHM gaussian pulse. The hohlraum was heated very asymmetrically by using only the lower half of Shiva's 20 beams, as show in figure la (Shiva had a vertical axis of symmetry). The resulting, time integrated thin wall images are shown in figure $1 \mathrm{~b}$, for an empty hohlraum, and figure $1 \mathrm{c}$ for a hohlraum with a capsule supported by a $1000 \mathrm{~A}$ thick formvar membrane. Figure $1 \mathrm{~b}$ showed, for the first time, that in an empty hohlraum the beams will go where we aim them. Quantitative analysis indicated that is was consistent with high absorption and geometric optics [5]. In particular, it ruled out scenarios where there would be a very large scattering of laser light throughout the hohlraum. Figure $1 \mathrm{c}$, from the hohlraum with the capsule and $1000 \mathrm{~A}$ support membrane, provided a surprise. Quantitative analysis of this image [5] indicated that while most of the energy strikes the upper wall, a significant fraction of the energy, $\sim 10 \%$ of the 1 micron laser light, appears to have been specularly reflected by the $1000 \mathrm{~A}$ formvar membrane. The time integrated image provided no information about the timing of the reflection. If it is an early time phenomena, as it presumably is, it would represent a sizable fraction of the incident 1 micron power being specularly reflected by a1000A membrane. Since Shiva was decommissioned shortly after these experiments we were unable to further explore this surprising, and still unexplained, finding. 
Thin wall imaging experiments were also among our earliest Nova hohlraum characterization shots $[6,7,8]$. Figure 2 is a montage of thin wall image data from a Nova hohlraum together with a clarifying, artists drawing of the hohlraum, laser beams and support stalk. The hohlraum was 1600 microns in diameter, 2750 microns long with laser entrance holes (LEHs) that were $75 \%$ of the hohlraum diameter. The hohlraum was made of tungsten of 2 microns thickness fabricated by Los Alamos National Laboratory. We irradiated it with an $\sim 16 \mathrm{~kJ}, 1 \mathrm{~ns}$ FWHM flattop (temporal shape) pulse of blue $(0.35 \mathrm{~nm})$ laser light. The image was taken with a time integrating, xray pinhole camera located at $68^{\circ}$ polar angle relative to Nova's axis of symmetry. This off-equatorial view causes the spots to separate via parallax, thus allowing us to identify all 10 of Nova's spots. However, because the hohlraum wall provides most of the spectral filtering, the emission from the laser entrance hole is at a considerably lower photon energy than the spot emission.

The interpretation given to figure 2 when it was first taken in 1986 is that the laser goes where we aim it. Quantitative analysis of the spots showed the center to center spacing to be $1600+-100$ microns, and the rms displacement from where we tried to aim the beams to be 80 microns.

The data of figures $1 \mathrm{~b}$ and 2 provided the first evidence that the laser beams in a hohlraum go where you aim them [9]. Such images have been popularly interpreted as a demonstration of very high first bounce absorption. Analysis allows us to use the images to set 
quantitative lower bounds on the absorption. The first step in this analysis is to write down expressions for the ratio of laser intensity due to scattered (i.e. unabsorbed) light ( $\left.I_{w a l l}\right)$ to the laser intensity striking the spot $\left(\mathrm{I}_{\text {spot }}\right)$.

$$
\begin{aligned}
& I_{\text {wall }} / I_{\text {spot }}=(1-\text { absorption }) A_{\text {spot }} /\left(\text { absorption } * A_{\text {wall }}\right) \\
& \text { or } \\
& I_{w a l l} / I_{\text {spot }}=(1-\text { absorption }) A_{\text {spot }} / A_{\text {wall }}
\end{aligned}
$$

In these expressions $A_{\text {spot }}$ and $A_{w a l l}$ are, respectively, the areas of the spot and the area of the wall illuminated by the scattered light. In a typical Nova hohlraum $A_{\text {wall }}$ can be as big as $6 A_{\text {spot }}$. The difference between equations [2] and [3] is that [2] assumes that processes which reduce absorbtion occur in the beampath before the laser energy can contribute to spot brightness. [3] assumes that scattering processes occur at the hot spot and that the full laser power contributes to spot brightness. The next step in absorption analysis is to consider figure 3 which plots $>6 \mathrm{keV}$ exposure vs laser intensity. This plot comes from Lasnex simulations of $0.35 \mu \mathrm{m}$ laser light incident upon gold. If we assume that the greatest dynamic range we can see in the data is about a factor of 20 , then the curve of figure 3 implies that the largest laser intensity ratio we can measure is about a factor of 10 (A dynamic range of 20 is consistent with the data of figures 1 and 2). However, since the wall emission we see comes from both the near and far wall while the spot emission comes from only one side, the factor of 10 becomes $I_{w a l l} / I_{\text {spot }}$ of $1 / 15$, as indicated in the figure. Using $A_{\text {spot }} / A_{w a l l}$ of 6 and $I_{w a l 1} / I_{\text {spot }}<1 / 15$ 
gives absorption $>70 \%$ with equation [2] and $>60 \%$ with equation [3]. Thus images like figures 2 , while indicating good absorption, provide room for up to $\sim 30-40 \%$ isotropic scattering of the incident energy. Further constraints on absorption would require auxillary measurements or different techniques.

As we discussed earlier, thin wall imaging of a pure Au hohlraum actually provides a picture of the temperature-density function of equation 1. In a gas filled or low- $Z$ lined hohlraum it measures the same function, but only for the plasma that is gold. For short pulses, when the hohlraum blow-off is similar to a disc's, emission of equation 1 coincides with the laser deposition. However, with a sufficiently long pulse, hot plasma will accumulate in a hohlraum, even in places well outside the beam path. Consequently, images of quantity [1] can be very different from "images" of laser deposition. Figure $4 a$ and $b$ show how readily thin-wall imaging can differ from laser deposition. Figure $4 \mathrm{a}$ shows $>6 \mathrm{keV}$ emission/cc from a Lasnex simulation of a Nova hohlraum driven by a $2.2 \mathrm{~ns}, 4: 1$ contrast ratio pulse shape known as ps26 [9]. The image is taken at $2 \mathrm{~ns}$ into the pulse, a time when the hohlraum is relatively filled with plasma. Experimentally, we could produce information like figure $4 \mathrm{a}$ by abelinverting a thin-wall imaging snapshot taken at $2 \mathrm{~ns}$ (after correcting for increasing wall attenuation at large radii because of the limb effect). Figure $4 \mathrm{~b}$ is a map of laser deposition/cc at $2 \mathrm{~ns}$ in the same simulation. It is very different from the $>6 \mathrm{keV}$ emission/cc. For our ps26 simulations we find that there is a good correlation between deposition and thin wall emission up to $\sim \operatorname{lns}$. After that the 
correlation is quickly lost. The conclusion we draw from this comparison is that while thin wall images may be a good indicator of laser deposition at early times, at later times, when the hohlraum fills with hot plasma, it becomes an unreliable measure of deposition. Ultimately, thin-wall imaging measures the quantity of equation 1 and not some other, more useful quantity, such as laser deposition or thermal $\mathrm{x}$-ray emission.

While thin-wall images are not a reliable measure of laser deposition at late times, we have used them to gain insight into late-time bulk plasma conditions. Figure $1 \mathrm{~b}$ and, later, figure 2 provided the seminal evidence of interpenetrating plasma in laser heated hohlraums. In a 2-D fluid hydrodynamics code like Lasnex, inwardly moving blow-off necessarily stagnates on the cylindrical axis of symmetry. The $>6 \mathrm{keV}$ emission/cc from this stagnated blow-off is large because it is hot and dense, see equation 1. The large axial emission in the simulation of figure $4 \mathrm{a}$ is a result of this effect. Our fluid simulations indicate that for many situations, such as $1 \mathrm{~ns}$ square top experiments, the stagnated, axial plasma emission should actually dominate time integrated images. The fact that only a very weak axial image is seen in images such as figures $1 \mathrm{~b}$ and 2 , is attributed to the interpenetrating nature of hot plasmas of sufficiently low density $[10,11,12,13,14]$. Hot, low-density plasmas have a collisional mean-free paths which are longer than the stagnation scale in Nova hohlraums. 
Thin wall imaging also provided the first evidence that Lasnex can model when a hohlraum fills to a significant fraction of critical density. We performed an experiment using a small, scale 0.625 hohlraum (1000 microns diameter, 1700 microns long). The hohlraum was irradiated with a "picket-fence" pulse which had three 100 ps wide pulses at $0,2.2$ and $3.5 \mathrm{~ns}$, followed by $1 \mathrm{~ns}$ flattop main pulse. The data indicated that the earliest pulse gets in and deposits at the wall. However during the main pulse the emission comes largely from the region near the laser entrance hole. This finding was consistent with our modelling which said that this small hohlraum would be filled with plasma at $4 \mathrm{~ns}$.

The experiments and analysis of thin-wall hohlraums we have discussed was performed in the 1980's while many aspects of the US Inertial Confinement Fusion program were still classified. Since then Los Alamos National Laboratory has taken the lead in thin wall hohlraum experiments. They have been using thin-wall imaging to measure beam pointing, as part of Precision Nova $[15,16]$ work; to image capsule implosion shapes without perturbing diagnostic holes [17] and to infer displacements in early time and, possibly, late time laser produced hot-spots in gas filled hohlraums [18].

This work was performed under the auspices of the U.S. Department of Energy by the Lawrence Livermore National Laboratory under Contract No. W-7405-ENG-48. 
References:

1- E. M. Campbell, Laser Part. Beams 9, 209 (1991).

2- D. E. Post, R. V. Jensen, C. B. Tarter, W. H. Grasberger, and W. A.

Lokke, Atom. Data Nucl. Data Tables 20, 397 (1977).

3-G. B. Zimmerman and W. L. Kruer, Comments Plasma Phys.

Controlled Fusion 2, 51 (1975).

4-R. Godwin, et. al., Laser Program Annual Report-1976, UCRL$50021-76,2-54$ (1976).

5- F. Ze, L. J. Suter, R. H. Price and C. L. Wang, Laser Program Annual Report 81/82, UCRL-50055-81/82, 4-43.

6- R. Kauffman, F. Ze, et. al., 1986/87 Laser Program Annual Report, UCRL-50055-86/87, 274.

7-A. R. Thiessen, 1986/87 Laser Program Annual Report, UCRL$50055-86 / 87,46$.

8- R. L. Kauffman, L. J. Suter, C. B. Darrow, J. K. Kilkenny, H. N. Kornblum, D. S. Montgomery, D. W. Phillion, M. D. Rosen, A. R. Thiessen, R. J. Wallace and F. Ze, Phys. Rev. Lett. 73, 2328 (1994).

9.- C. J. Keane, private communication (LLNL, 1993).

10- J. Albritton and C. Randall, private communication (LLNL, 1983). 11 - R. A. Bosch, R. L. Berger, B. H. Failor, N. D. Delameter, G. Charatis, and R. L. Kauffman, Phys. Fluids B 4, 979-988 (1992).

12- S. M. Pollaine, R. L. Berger and C. J. Keane, Phys. Fluids B 4,

13- L. J. Suter, 986/87 Laser Program Annual Report, UCRL-50055$86 / 87,54$.

14- M. Jones, private communication (Los Alamos National Laboratory, 1993). 
15- 1994 ICF Annual Report (UCRL-LR105820-94). The first quarter's report was entirely devoted to Precision Nova. 16- D. Baker, et. al. Bull. Am. Phys. Soc. 38, 2083 (1993). 17- A. Hauer, N. Delameter, D. Ress, et. al., Rev. Sci. Instrum. 66, 672 (1995).

18- N. Delameter, et. al. , Physics of Plasmas, May, 1996 (in press).

Figures:

1-a) $2.5 \mathrm{~mm}$ diameter thn wall Shiva hohlraums were irradiated by the lower beams only. This figure also shows a capsule and $1000 \mathrm{~A}$ formvar support membrane. b) Thin wall image for an empty Shiva hohlraum. c) Image for a hohlraum with capsule and $1000 \mathrm{~A}$ formvar support membrane.

2- Montage of thin wall data from a standard Nova hohlraum and a drawing of the hohlraum, laser beams, and support stalk. The principle features of the thin wall data are the spots, the weak emission along the axis, and the unfiltered emission from the laser entrance hole.

3-Lasnex estimates of $>6 \mathrm{keV}$ emission vs. laser intensity for a 0.35 micron laser.

4- Contour plots of $>6 \mathrm{keV}$ emission/cc (a) and laser deposition (power/cc) (b) from a simulated Nova hohlraum, after it has filled with plasma. $5 \%, 10 \%, 25 \%, 50 \%, 75 \%$ and $90 \%$ contour values. 
We only plot $1 / 4$ of the full view since the simulated hohlraums are left-right symmetric about the midplane and rotationally symmetric about the z-axis. 


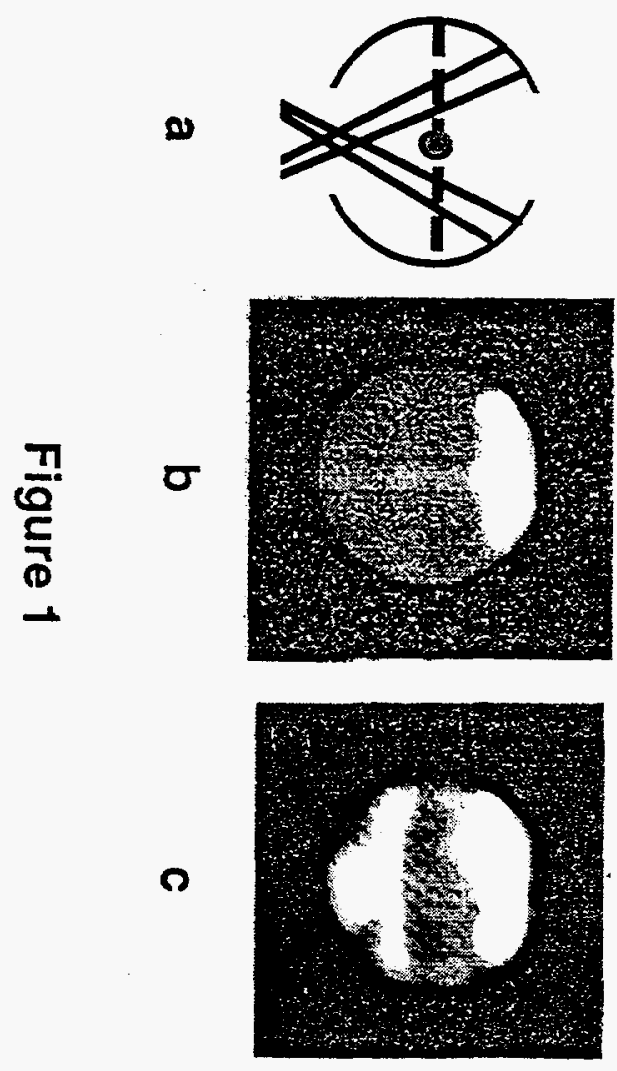




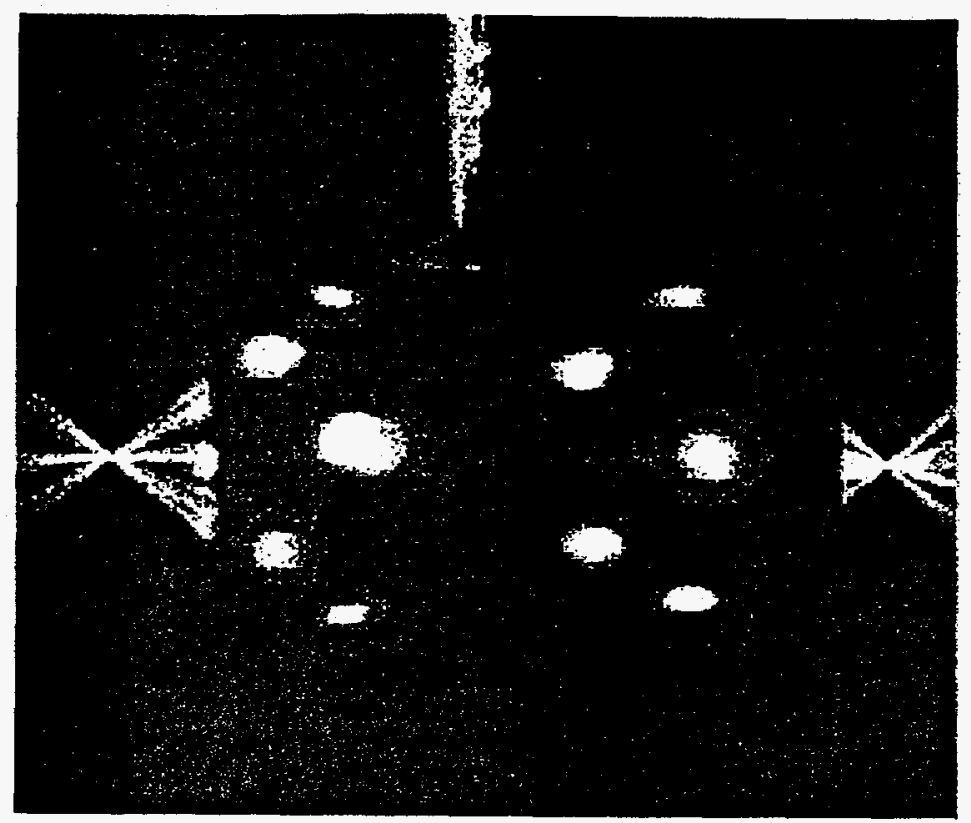

Figure 2 
$\frac{\pi}{\frac{1}{6}}$

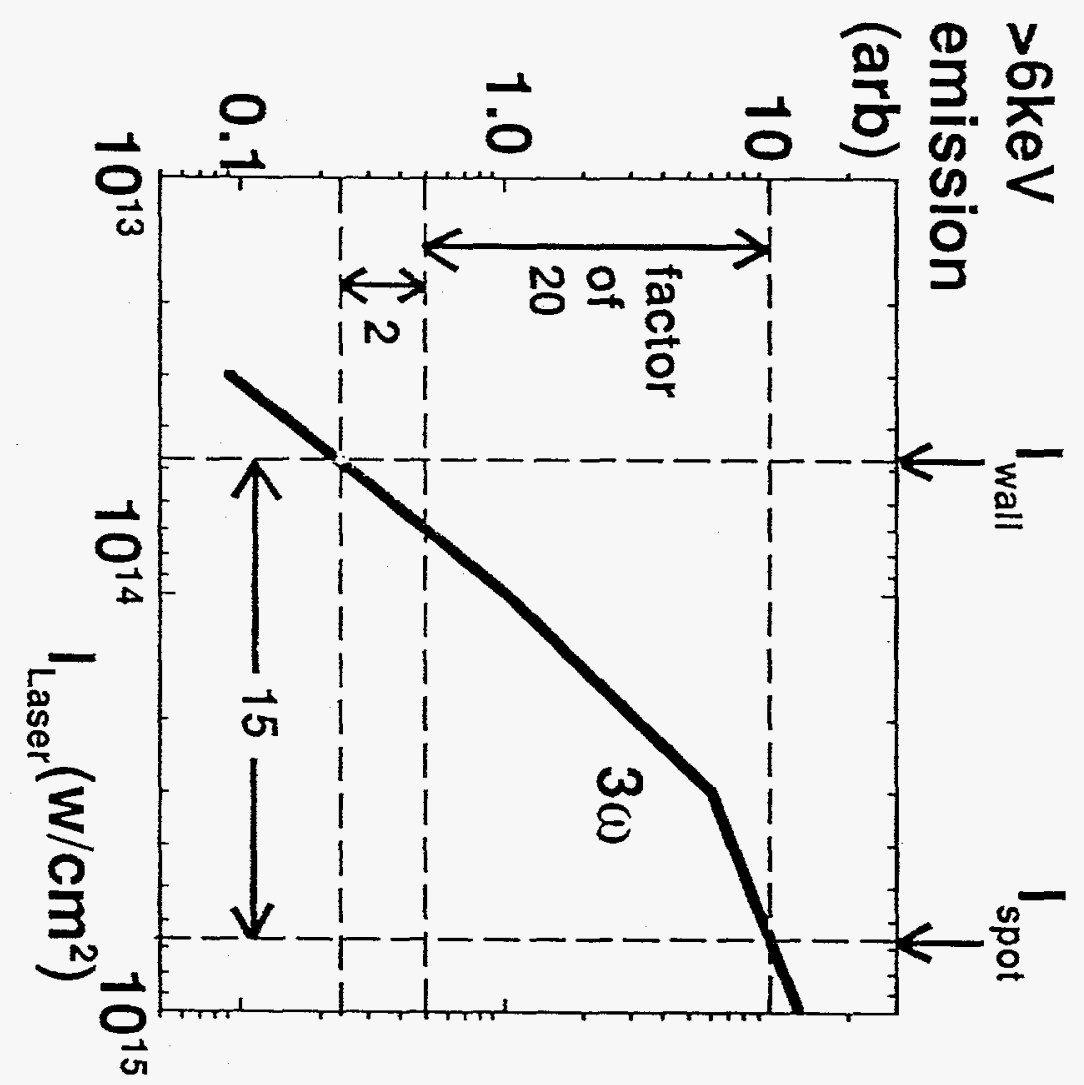


dj4568 >6kev emission/cc at. 200

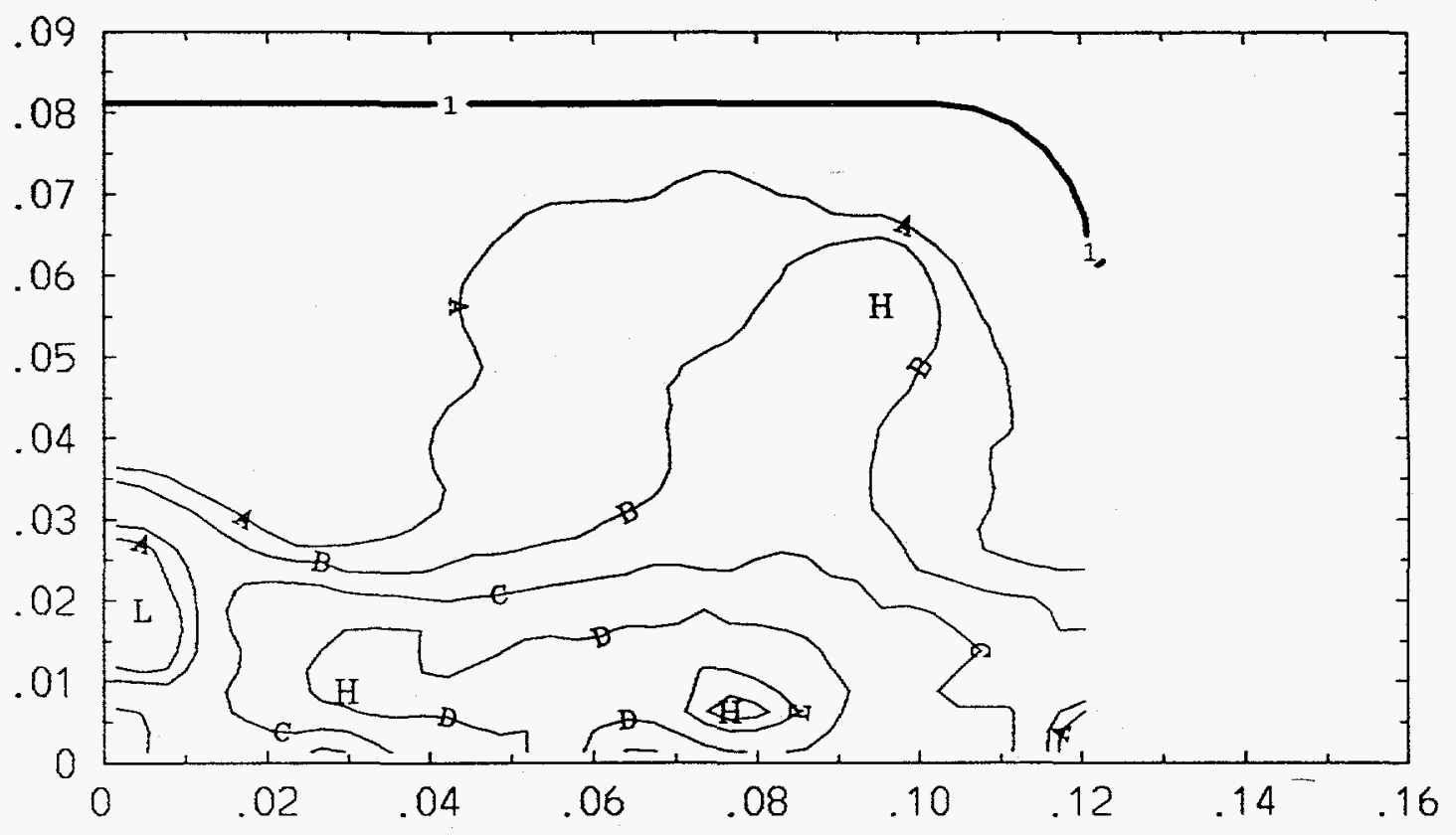

1: plot fart fqzt thick $=3$

plotc pict xmesh ymesh lev=zcontours

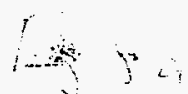


dj4568 at .200

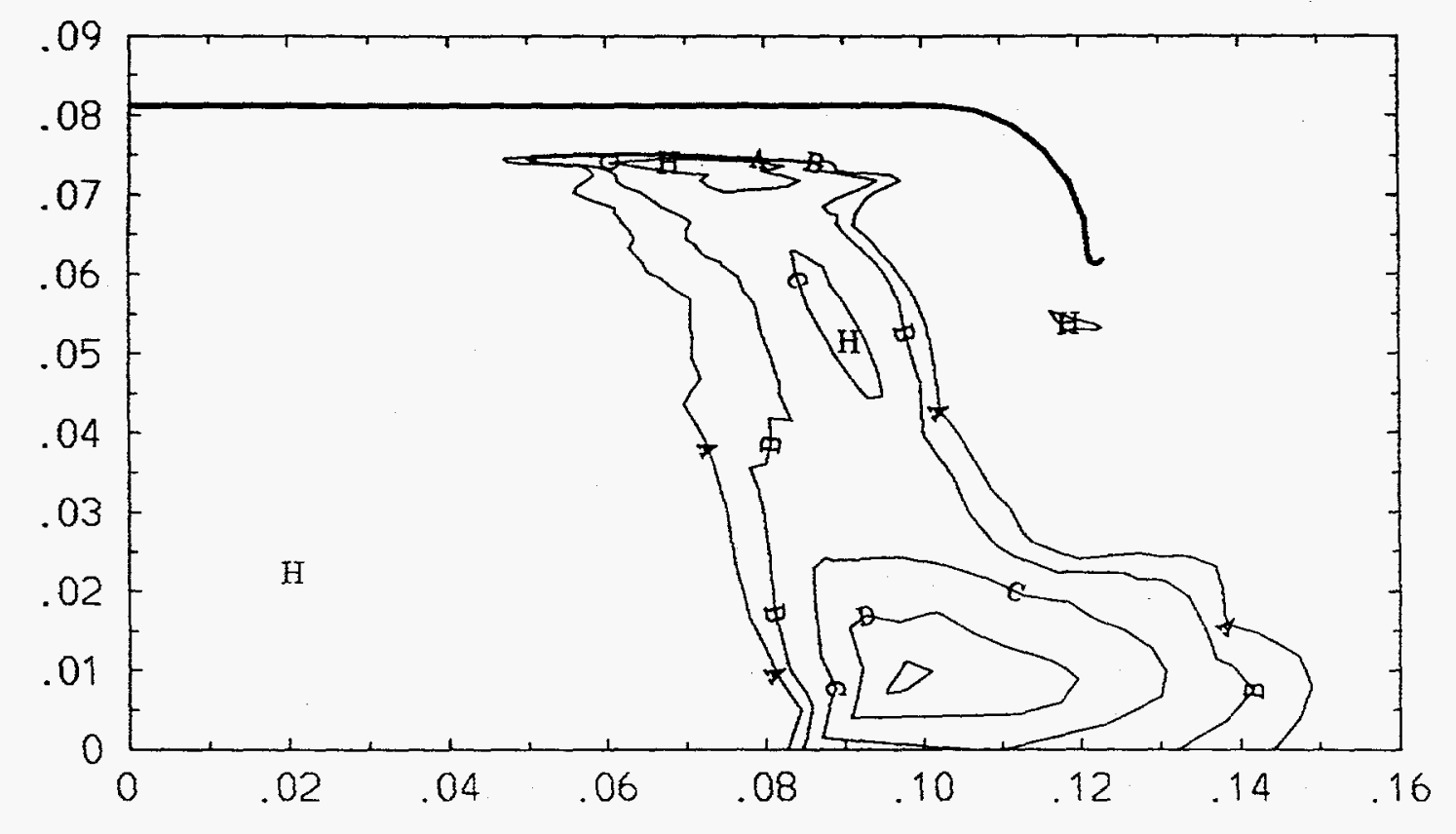

plotc el_v lev=zcontours

plotm Irange $=1$ max thick $=3$
A $3.218 E-02$

B $6.435 \mathrm{E}-02$

C $1.609 \mathrm{E}-01$

D 3.218E-01

E 4.826E-01

F 5.792E-01 